\title{
News from ASCO 2015 - What Was Relevant for Breast Cancer Experts?
}

\author{
Chair: Jens Huober ${ }^{a}$ \\ Participants: Thomas Decker ${ }^{b} \quad$ Cornelia Liedtke $^{c} \quad$ Frederik Marméd $^{d} \quad$ Norbert Marschner $^{\mathrm{e}}$ \\ Volkmar Müller ${ }^{f}$ \\ a Frauenklinik, Universitätsklinikum UIm, Germany; \\ b Onkologie Ravensburg, Ravensburg, Germany; \\ ${ }^{c}$ Klinik für Frauenheilkunde und Geburtshilfe, Universitätsklinikum Schleswig-Holstein / Campus Lübeck, Germany; \\ d Nationales Centrum für Tumorerkrankungen (NCT) Heidelberg und Universitätsfrauenklinik Heidelberg, Germany; \\ ${ }^{e}$ Gemeinschaftspraxis für interdisziplinäre Onkologie und Hämatologie, Freiburg i.Br., Germany; \\ ${ }^{f}$ Department of Gynecology, University Medical Center Hamburg-Eppendorf, Hamburg, Germany
}

\section{Question 1: What Were Your Highlights at this Year's ASCO?}

Decker: Phase III data of the PALOMA3 trial were presented in the oral abstract session. These data convincingly confirmed a statistically significant and clinically relevant prolongation of progression-free survival (PFS) that has been previously published in a phase II trial. In patients with metastatic hormone receptor-positive (HR+), HER2-negative (HER2-) breast cancer with progressive disease after aromatase inhibitor (AI) therapy, PFS was prolonged from 3.8 to 9.2 months when the CDK4/6 inhibitor palbociclib was added to fulvestrant. Toxicity was only mildly increased (neutropenia) as previously reported in the PALOMA2 trial. Importantly, premenopausal women were also included in this trial (GNRH analogues were added in this patient population) and had the same degree of benefit as postmeopausal women.

Liedtke: To my personal opinion, there are several answers to this question. First, from a clinician's point of view the positive results presented by Turner and colleagues regarding the PALOMA3 study were the most clinically relevant results. In this trial the addition of palbociclib to the standard hormonal therapy fulvestrant more than doubled the duration of disease control, resulting in a delay of disease progression of roughly 5 months in women with pretreated, HR+/HER2- metastatic breast cancer. Although registration of palbociclib in Europe is still pending, these results together with the PALOMA1 trial (in which palbociclib was added to letrozole) will soon provide an important and efficacious option for the treatment of $\mathrm{HR}+$ metastatic breast cancer.
As an investigator of the ADAPT trial was glad to see the results of the ADAPT HR+/HER2+ study had been accepted for oral presentation. In this trial a pathologic complete response (pCR) rate of $45.8 \%$ could be achieved with trastuzumab-DM1 (T-DM1) in combination with endocrine therapy. These results are particularly important given the very low toxicity profile of this treatment arm, in which patients had not yet been treated with anthracycline-taxane containing combination chemotherapy.

From a more general perspective I am amazed in face of the large body of evidence accumulating with regard for immunotherapy in various cancer entities. For instance, one study compared use of the anti-PD1-antibody nivolumab to chemotherapy demonstrated a significant improvement in overall survival (OS) with fewer side effects for patients with advanced lung cancer. Another trial used a combination of different immunotherapeutic agents (nivolumab and ipilimumab) for the treatment of patients with advanced melanoma. Combination therapy resulted in significantly higher response rates when both therapies were used in combination compared to ipilimumab alone. Several trials in which these agents are used for the treatment of (triple negative) breast cancer are currently ongoing or being initiated.

Müller: The oral presentation of a first interim analysis from the ADAPT trial with neoadjuvant therapy for 'triple-positive' (e.g. HER2+ and $\mathrm{HR}+$ ) patients. In an oral presentation, results from the first 130 patients were presented (abstract 507). Patients were treated for 12 weeks prior to surgery with either A: T-DM1, B: T-DM1 plus endocrine therapy (tamoxifen for premenopausal or aromatase inhibitor (AI) for postmenopausal women) or C:

\section{KARGER}

Fax +497614520714
(๑) 2015 S. Karger GmbH, Freiburg

$1661-3791 / 15 / 0104-0285 \$ 39.50 / 0$
Prof. Dr. Jens Huober 
trastuzumab plus endocrine therapy. pCR rates were A: $40.5 \%, \mathrm{~B}$ : $45.8 \%$, C: $6.7 \%$. The difference between either arm A or B vs. C was significant, but not A vs. B. This demonstrates for the first time clinically meaningful pCR rates (> 40\%) after only 12 weeks of T-DM1 \pm endocrine therapy without systemic chemotherapy in triple-positive breast cancer and might open a new perspective for less toxic treatment in these patients. However, a comparison with neoadjuvant pertuzumab and trastuzumab combined with chemotherapy is pending. In this context of HER2+ breast cancer, 2 other presentations with follow-up data from neoadjuvant trials were of relevance: Data from the HannaH trial showed a correlation between $\mathrm{pCR}$ and improved outcome in patients treated with trastuzumab (either i.v. or subcutaneous) in $\mathrm{HR}+/ \mathrm{HR}$ - patients (abstract 585). Moreover, follow-up data from the neoadjuvant trial Neosphere was presented (abstract 505). Here, the enhanced pCR rate of adding pertuzumab to trastuzumab combined with chemotherapy resulted in an improved disease-free survival for the combination.

\section{Question 2: Were There 'Practice Changing' Presentations which Will Have Direct Impact on Your Clinical Practice Following ASCO?}

Decker: The PALOMA3 trial adds level-1 evidence for the combination of CDK4/6 inhibitors and antihormonal treatment and will very likely result in a full approval of palbociclib in combination with antihormonal treatment in patients with metastatic $\mathrm{HR}+/$ HER2- disease.

Liedtke: Again, I would refer to the PALOMA3 trial at this point. However, since palbociclib is not yet registered these results will not influence treatment tomorrow but in the very near future.

Marmé: Nicholas Turner presented data from PALOMA3 (LBA 502). The addition of palbociclib lead to an improvement in PFS from 3.8 to 9.2 months (HR 0.42, p $<0.00001$ ). Palbociclib has been granted accelerated approval in combination with letrozole in 1st line treatment of metastatic breast cancer by the FDA, based on the data from PALOMA1. PALOMA3 not only confirms the efficacy of palbociclib but offers data on premenopausal women, who have been neglected in endocrine phase III trials in the last decades. Importantly, the toxicity profile of palbociclib appears very favorable, with neutropenia being the most prominent and easily manageable toxicity. As we are still awaiting regulatory approval of palbociclib, there are numerous CDK4/6 inhibitor trials recruiting in Germany and patients can already benefit.

Marschner: I was impressed by the presentation of Gajra et al. on 'Predictors of chemotherapy dose reduction at first cycle in patients age 65 years and older with solid tumors'. The presentation gives evidence that guidelines, derived solely from randomized controlled trials (RCTs), are often not suitable for real-life situations. The patient population in RCTs is highly selected and not predictive for patient groups differing in relevant patient-derived or clinical factors. There has to be a 'rethinking' for the preparation of guidelines.

\section{Question 3: Were There any Abstracts With the Potential to Influence Future Developments and to Guide Future Trial Concepts?}

Decker: The exciting field of immunotherapy with oral presentations reporting the success of checkpoint inhibitors in hard-totreat cancers like non-small cell lung cancer and melanoma will hopefully stimulate research in triple negative breast cancer.

Nadia Harbeck presented data of the ADAPT trial. This randomized trial with 3 treatment arms showed high efficiacy and good tolerability of neoadjuvant treatment with single-agent T-DM1 for 12 weeks in patients with triple-positive breast cancer. Stringent pCR was achieved in $40 \%$ of treated patients - much better than the reported pCR rate of $6 \%$ when patients were treated with trastuzumab and endocrine treatment. However, results were only marginally better when T-DM1 was combined with endocrine treatment. Results with single-agent T-DM1 seemed to be superior in postmenopausal women.

Data from the DETECT III trial showed that circulating tumor cells (CTCs) could be detected in $60 \%$ of patients with metastatic breast cancer. All patients had HER2- primary cancer. Importantly, at least one HER2 + cell was detected in 18\% of CTC-positive samples. This study demonstrates feasibility and clinical relevance of performing liquid biopsies in patients with metastatic breast cancer. The data of the clinical part of the trial (comparing standard treatment to treatment in combination with lapatinib in patients with HER2+ CTCs) are eagerly awaited.

Liedtke: There are several aspects that may be mentioned in this context. First, accumulating data regarding efficacy of immunotherapeutic agents in solid cancers justifies integration of these agents into clinical trials for breast cancer. Second, there is increasing evidence that biomarkers are needed for patient stratification, particularly with regard to targeted agents. Therefore, translational research programs are urgently needed to be integrated in any clinical trial to allow for development of appropriate companion diagnostics. Third, there seems to be an increasingly acknowledged fact that HER2+/HR+ and HER2+/HR- breast cancers are two entities with entirely distinct biology. Clinical trials have to address this and offer concepts that specifically address questions for these two entities.

Marmé: Nadia Harbeck presented first data on pCR rates in the HER2+/HR+ cohort of the ADAPT trial (abstract 506). Patients in the T-DM1 containing arms achieved pCR rates of 41 and $46 \%$, respectively compared to $7 \%$ in the trastuzumab containing arm. The pCR rates on T-DM1 are the more intriguing as patients only received 4 cycles and had $\mathrm{HR}+$ tumors, in which pCR rates are significantly lower compared to HER2+/HR- tumors. There are still 
many open questions for consideration in future trials, such as the role of dual HER2 targeting in the setting of T-DM1, but the data suggest that there is a promising way to de-escalate therapy in HER2-positive breast cancer. The trial also comprises a comprehensive biomarker program. Hopefully, this will lead to the identification of patients who may not need a full standard combination chemotherapy in addition to their HER2-targeted therapy.

Complex but important data on the predictive and prognostic role of PIK3CA mutations in HER2+ early breast cancer were presented by Sibylle Loibl. A combined analysis of nearly 1,000 patients from 3 prospective clinical trials, confirmed a PIK3CA mutation rate of $22 \%$ in HER2+ early breast cancer and also confirm that PIK3CA mutations confer a significantly lower pCR rate. This was predominantly seen in tumors which were also $\mathrm{HR}+$. In fact, in this subgroup the pCR rate was only $7.6 \%$ despite HER2 targeted therapy. In the overall population PIK3CA did not impact PFS, however, there was a positive interaction between mutation status and HR status. In HR+ patients PFS was shorter in PIK3CA mutation carriers whereas it tended to be longer in in HR- patients. Thus PIK3CA status might identify a subgroup of patients with a low probability of $\mathrm{pCR}$ and a poor prognosis in HER2+ early breast cancer, which might warrant investigation of PI3K targeted agents or other experimental strategies.

Homologous recombination (HR) deficiency assays are currently in the focus to identify patients with HR deficiency beyond BRCA mutations which might benefit from platinum-based chemotherapy or PARP inhibitors. Diverse tests are being evaluated, based on different genomic alterations, such as LOH, LST, TAI, or specific amplifications and deletions. BRCA mutation status is a predictive factor for platinum sensitivity in the GeparSixto trial. This year Gunter von Minckwitz presented the data on HR deficient tumors based on an HR deficiency score and deleterious tumor BRCA mutations. Combining these data, roughly $70 \%$ of patients were predicted to have a HR deficiency, whereas germline BRCA mutations could only be demonstrated in about $13 \%$ (ASCO 2014). HR deficiency significantly predicted pCR, however, this was irrespective of therapy and there was no interaction between treatment and HR deficiency. Results from several exploratory subgroup analyses remain inconclusive and validation in independent trials is needed. Similarly in triple negative tumors the same HR deficiency score failed to predict platinum benefit, whereas germline BRCA mutations were a significant predictor. Further, this year's meeting demonstrated our growing insight into the complex issue of tumor infiltrating lymphocytes and neoantigens as possible predictors for immune checkpoint inhibition strategies.

Marschner: I think the presentation of Fried et al. 'Understanding the treatment preferences of seriously ill patients' highlighted very nicely that patient reported outcomes (PRO) should be paid much more attention in the future. The focus only on efficacy parameters is no longer sufficient for the evaluation of treatments or procedures.
Müller: Besides the neoadjuvant trials mentioned above, many presentations about immunotherapeutic approaches in other tumor entities, such as melanoma and lung cancer, were exciting (e.g. abstracts LBA 1, LBA 109). As indicated by small pivotal trials in breast cancer patients that were presented at the San Antonio Breast Cancer Symposium 2014 and at the AACR meeting this year, this hope might also be fulfilled for breast cancer.

\section{Question 4: Were There Any Disappointing Presentations?}

Decker: The phase III MARIANNE trial has dampened hopes that dual HER2 treatment with T-DM1 and pertuzumab can further improve the treatment of patients with Her2+ metastatic breast cancer. Although T-DM1 was as effective as trastuzumab combined with a taxane, combination treatment with pertuzumab and T-DM1 was not more efficacious. This negative result had immediate implications for major ongoing adjuvant trials exploring this combination.

A large effort was made to compare 3 different bisphosphonates in adjuvant treatment of early breast cancer. More than 5,000 patients were randomized to receive zoledronate, clodronate, or ibandronate. The results of the S0307 trial demonstrated no difference in DFS or overall survival. Minor differences in toxicities were noted. This study was disappointing because it wasted a lot of resources for a question of minor scientific and clinical relevance.

Liedtke: One important clinical question is the optimal use of platinum-containing agents for treatment of triple negative breast cancer. There is a growing body of evidence that BRCA dysfunction might be associated with increased response to platinumcontaining chemotherapy. However, there is yet no consistent data on how to best identify lack of BRCA function relevant for patient stratification. von Mickwitz et al. presented translational results of the GeparSixto trial and evaluated the association between response to platinum-based chemotherapy and (i) genetic BRCA mutation status (ii) tumor BRCA mutation status and (iii) HR deficiency as a measure for BRCAness. BRCA dysfunction was associated with increased sensitivity regarding both platinum-based and platinum-free chemotherapy, however, no significant association between use of carboplatin and increase in pCR was seen. Therefore, BRCA dysfunction in this analysis came out as biomarker for overall chemotherapy sensitivity rather than a platinum-specific biomarker. Therefore, the search for the optimal biomarker for stratifying patients to platinum-based chemotherapy or not is not yet finished. Until additional results will be available, German national guidelines suggest use of platinum (in the neoadjuvant or metastatic setting) based on BRCA mutation status $(\mathrm{AGO}+)$.

Marmé: Of course neither the presentation nor the study design of the MARIANNE trial were disappointing. However, the results 
of the primary endpoint did not meet our hopes. After several years of milestone improvements in HER2+ breast cancer, most of us had hoped that MARIANNE would fit in this series. However, this trial failed to demonstrate any improvement, either for T-DM1 over trastuzumab or for the addition of pertuzumab to T-DM1 in the first-line metastatic setting. Therefore these data will not change our treatment algorithm even if T-DM1 may offer an effective and less toxic treatment option in this setting.

Müller: The results of a translational research project from the neoadjuvant GeparSixto trial did not fulfill my hopes, although I would not call it disappointing (abstract 1004). This project tried to improve the prediction of $\mathrm{pCR}$ to carboplatin-based neoadjuvant therapy using HR deficiency in patients with triple negative breast cancer. Background is the lack of predictive factors for the use of carboplatin-based therapy in the neoadjuvant setting. This analysis in my opinion did not demonstrate a clear predictive value of $\mathrm{HR}$ deficiency, although HR deficiency predicted increased response rates to chemotherapy in general.

\section{Participants}

Professor Dr. med. Thomas Decker

Onkologie Ravensburg

Elisabethenstraße 19, 88212 Ravensburg, Germany

decker@onkonet.eu

PD Dr. med. Cornelia Liedtke

Klinik für Frauenheilkunde und Geburtshilfe,

Universitätsklinikum Schleswig-Holstein / Campus Lübeck

Ratzeburger Allee 160, 23538 Lübeck, Germany

cornelia.liedtke@uksh.de

PD Dr. med. Frederik Marmé

Nationales Centrum für Tumorerkrankungen (NCT) Heidelberg und Universitätsfrauenklinik Heidelberg

Im Neuenheimer Feld 460, 69120 Heidelberg, Germany

Frederik.Marme@med.uni-heidelberg.de

Dr. Norbert Marschner

Gemeinschaftspraxis für interdisziplinäre Onkologie und Hämatologie Breisacher Straße 117, 79106 Freiburg i.Br., Germany

norbert.marschner@onkologie-freiburg.de

Prof. Dr. med. Volkmar Müller

Department of Gynecology, University Medical Center

Hamburg-Eppendorf

Martinistrasse 52, 20246 Hamburg, Germany

vmueller@uke.de 